\title{
Néolithisation et néolithique ancien du Maroc
}

\section{Aziz Ballouche, Brahim Ouchaou et Abdelaziz El-Idrissi}

\section{OpenEdition}

Journals

Édition électronique

URL : https://journals.openedition.org/encyclopedieberbere/2727

DOI : 10.4000/encyclopedieberbere. 2727

ISSN : 2262-7197

\section{Éditeur}

Peeters Publishers

\section{Édition imprimée}

Date de publication : 5 octobre 2012

Pagination : 5499-5512

ISBN : 978-90-429-2718-6

ISSN : 1015-7344

\section{Référence électronique}

Aziz Ballouche, Brahim Ouchaou et Abdelaziz El-Idrissi, « Néolithisation et néolithique ancien du Maroc », Encyclopédie berbère [En ligne], 34 | 2012, document N47, mis en ligne le 15 décembre 2020, consulté le 17 février 2022. URL : http://journals.openedition.org/encyclopedieberbere/2727 ; DOI : https://doi.org/10.4000/encyclopedieberbere.2727

Ce document a été généré automatiquement le 17 février 2022.

(ㄷ) Tous droits réservés 


\title{
Néolithisation et néolithique ancien du Maroc
}

\author{
Aziz Ballouche, Brahim Ouchaou et Abdelaziz El-Idrissi
}

1 Au Proche-Orient et en Europe, les termes de « Néolithisation » et de « Néolithique » désignent généralement l'émergence et le développement de sociétés dont l'économie est fondée sur un système de production de biens de subsistance, lui-même fondé sur la maîtrise et la manipulation des ressources de la nature que sont les plantes et les animaux. Dans les régions actuellement étudiées au Maroc (fig. 1), seuls quelques sites, comme Kaf Taht el-Ghar (région de Tétouan), permettent une telle approche. Les études approfondies sur les faunes et les végétaux ne font que commencer et sont loin d'être systématiques, en raison, entre autres, de la mauvaise conservation des restes organiques dans de nombreux sites archéologiques. Ces conditions obligent donc à reconsidérer la présence de la céramique comme un marqueur décisif, depuis que celleci procure de nouveaux résultats, tant au Niger qu'au Mali, appuyés par des séries cohérentes de datations très anciennes (10e millénaire av. J.C.) et de nouvelles informations paléo-environnementales et comportementales d'une grande précision. En accord avec E. Huysecom (2006), nous pouvons considérer que la céramique, généralement bien conservée, est un outil pratique pour une approche typologique des sociétés néolithiques parce qu'elle témoigne d'une certaine emprise des hommes sur leur environnement et de nouveaux comportements. C'est pourquoi, nous prenons en considération ici, par pragmatisme, tous les sites ayant livré de la céramique. Cette perspective permet de proposer pour le Maroc une esquisse régionale provisoire de la néolithisation. Rappelons que ce postulat n'est pas retenu par C. Roubet ("Néolithisation: Introduction », N40) et Roubet et Kherbouche (N46) pour l'Algérie. Il ne l'est non plus par N. Aouadi et Y. Dridi, pour la Tunisie (N44), ni par B. Barich et E. de Faucamberge (N42, N43) pour la Libye. Pourtant, dans le sud de la péninsule Ibérique, Manen et al. (2007) pensent que, chaque fois que les matériaux organiques ont été conservés dans les plus anciens sites à céramique, on peut conclure à un Néolithique au sens économique du terme. Mais cette situation n'est pas généralisable. Il faut signaler que, pour le Rif oriental, apres avoir utilise le terme de Néolithique ancien dans ses premiers travaux, Linstädter (2008) révise sa position et désigne ce faciès 
d'Epipaléolithique à poterie pour les sites sans trace reconnue d'une économie de production. On rappellera encore les travaux initiaux conduits en Libye tassilienne par B. Barich (N42) qui avait déjà proposé cette terminologie (d'Epipaléolithique à poterie) avant la reconnaissance d'animaux domestiques.

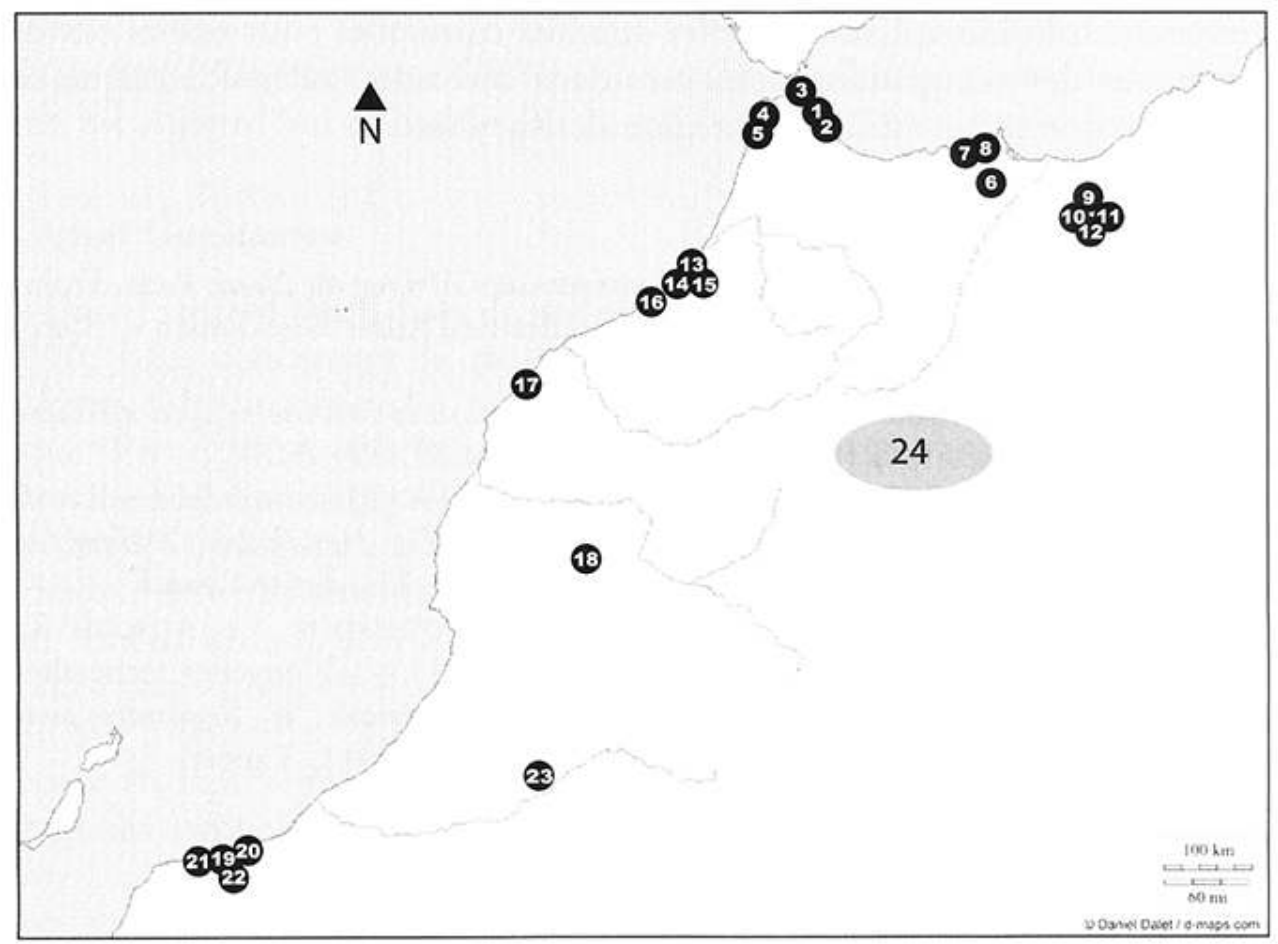

Fig. 1. Carte des principaux sites cités dans le texte : 1. Kaf Taht el-Ghar ; 2. Kaf Boussaria ; 3. Gar Kahal ; 4. Achakar (Idoles, El Khil, El Alya) ; 5. Tahadart ; 6. Hassi Ouenzga ; 7. Ifri Oudadane ; 8 . Ifri Armas ; 9 . El Heriga ; 10. Oued Isly ; 11. Grotte du Rhafas ; 12. Guenfouda ; 13. El Harhoura 2 ; 14. Grotte des contrebandiers; 15. El M'nasra ; 16. Skhirat; 17. Ma Izza ; 18. Toulkine; 19. Site $19 ; 20$. Izriten ; 21. Site Letan ; 22. Taoulekt ; 23. Adrar n'Metgourine (Akka) ; 24. Projet GENEMAR (Provinces de Figuig et Errachidia)

\section{Maroc septentrional}

\section{Néolithique de la péninsule Tingitane}

Plusieurs sériations du Néolithique de la péninsule Tingitane, fondées sur des études typologiques et stylistiques anciennes de la céramique, ont été proposées (Tarradell 1957, Jodin 1958-1959, Gilman 1975). La révision critique des collections des anciennes fouilles (Ghar Khal, Kaf Taht el-Ghar, Achakar) et l'étude des matériaux des nouvelles fouilles (Daugas 2002, Daugas et al. 1989, 1998, 2008, El Idrissi, 2001) ont permis un renouvellement du phasage du Néolithique de la péninsule Tingitane. Pour l'essentiel, la périodisation s'appuie sur les données du complexe Kaf Taht el-Ghar / Kaf Boussaria, complétées ensuite par les informations souvent partielles issues d'autres sites de la région (Tahadart, grottes d'El Khil, d'El Aliya, des Idoles...). Situées à une dizaine de kilomètres au sud de Tétouan, les deux grottes de Kaf Taht el-Ghar et Kaf Boussaria constituent les sites de référence pour le Néolithique ancien de la péninsule Tingitane. La première a été fouillée anciennement par M. Tarradell (1955), puis des travaux ont été repris dans le cadre d'un programme franco-marocain. La grotte voisine de Kaf Boussaria a été fouillée en 1995 et 1997 par une équipe de l'INSAP (El Idrissi 2001). Une 
lacune sédimentaire et des dépôts affectés d'importantes bioturbations mettent directement en contact les niveaux épipaléolithiques et néolithiques sans continuité. La séquence du Néolithique ancien, assise sur de nombreuses datations numériques croisées (tab. 1) se décline comme suit :

\section{Phase initiale}

Elle est représentée à Kaf Taht el-Ghar (KTG) et à Kaf Boussaria (KBS). Sa datation s'établirait au $7^{\mathrm{e}}$ millénaire av. J.C. La céramique (fig. 2) est caractérisée par des décors incisés (lignes verticales, motifs quadrillés et chevrons emboîtés) et des décors plastiques. L'impression de Cardium, signalée à KTG, est absente à KBS.
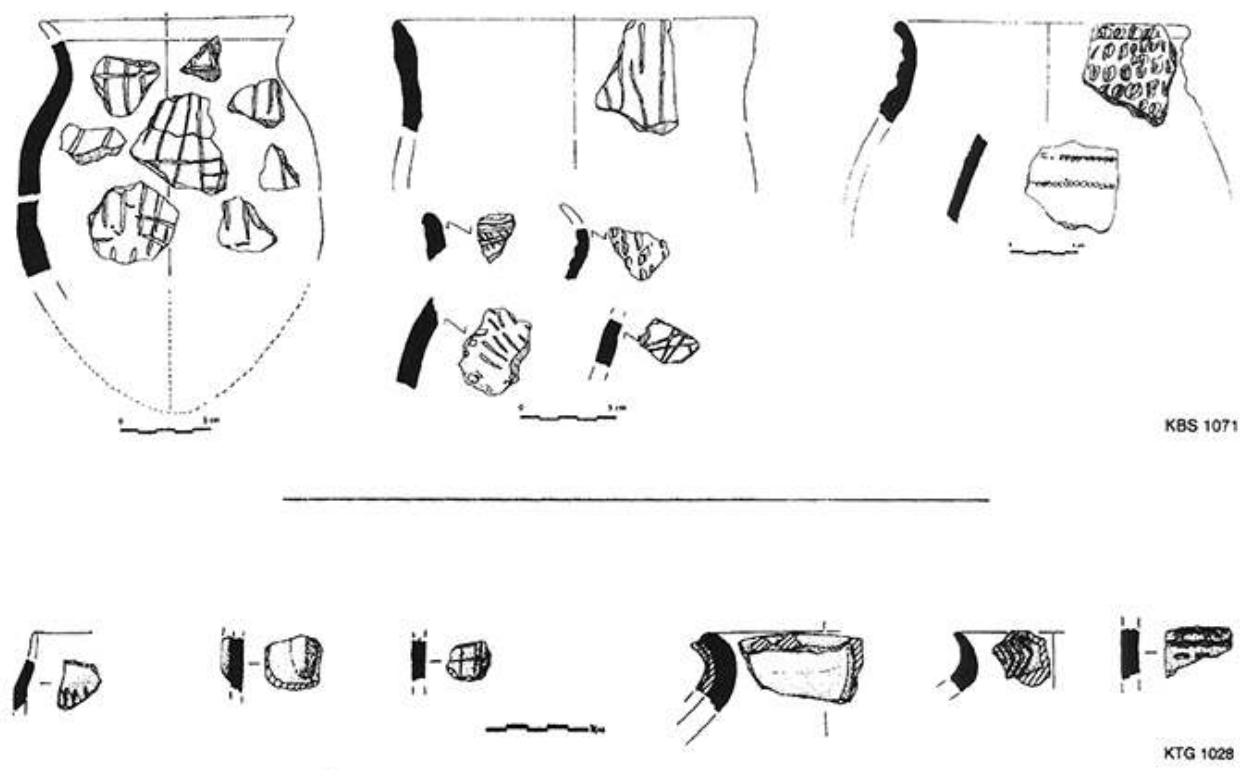

Fig. 2. CÉRAMIQUe de LA PHASE INITIALE dU NÉOLITHIQUE de KAF BOUSSARIA (US 1071) ET KAF TAHT EL-GHAR (US 1028) (D'APRÈS EL IDRISSI 2001).

Phase cardiale : se subdivisant elle-même en trois périodes.

6 - Période 1. Il s'agit d'une occupation sporadique, datée de la charnière 7e/6e millénaires av. J.C., qui correspond à l'apparition des premiers témoins du Cardial à décor cannelé ou d'impression à la coquille, dans un corpus céramique composé majoritairement de tessons lisses. L'industrie lithique, sur lames et lamelles produites par percussion directe, comprend des lamelles à dos, coches et denticulés.

7 - Période 2. Elle correspond à une occupation intensive au cours de la deuxième moitié du 6e millénaire av. J.C. Le site de KTG est aménagé : nivellement, sol en terre battue, empierrement, aires d'activités spécialisées. La céramique décorée par impression à la coquille de Cardium (impression souvent pivotante) est d'abord majoritaire en association avec d'autres décors imprimés ou plastiques et des cannelures. Les décors de cannelures deviennent ensuite prépondérants. Dans l'industrie lithique la percussion directe coexiste désormais avec un débitage par pression. 

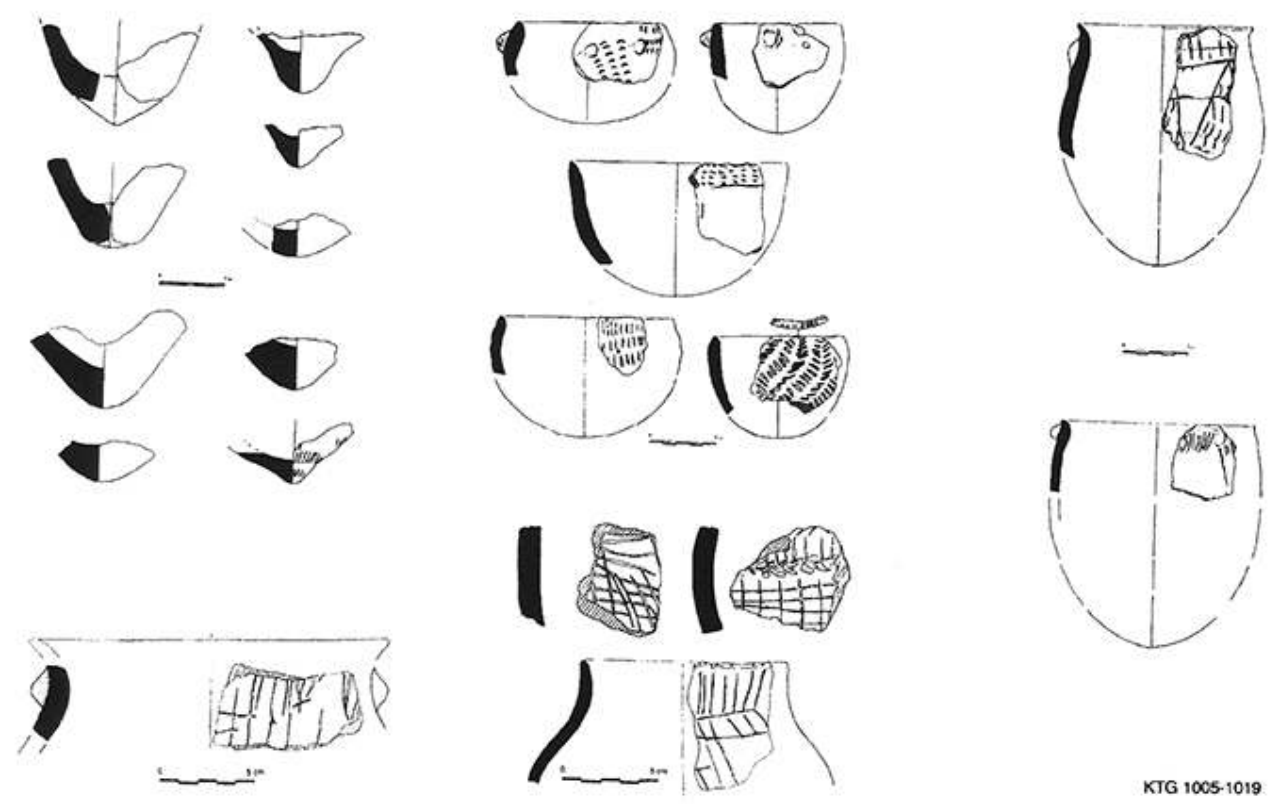

Fig. 3. CÉramique de LA deUXIÈme PHASE CARdiale de KAF tAHT el-GHAR (US 1005 et 1019) (D'APRÈS EL IDRISSI 2001).

- Période 3. En continuité avec la précédente, correspondant essentiellement à une évolution des décors vers un recouvrement grandissant des surfaces et une diminution du décor cardial s.s.

9 La dernière période documentée à KTG, correspond à partir du 5e millénaire av. J.C. à la généralisation des documents cardiaux et à une répartition géographique très large dans toute faire péninsulaire, à la fois méditerranéenne et atlantique, illustrée par les grottes d'El Khil, d'El Aliya, des Idoles, de Kaf Boussaria, de Ghar Kahal, le site de plein air de Tahadart. La poterie est d'une grande qualité technique, avec une syntaxe décorative élaborée qui apparaît structurée et complexe, et les motifs tendent à couvrir toute la surface visible du récipient (fig. 4). Cette richesse et cette diversité permettent d'ailleurs à Daugas et al. (2008) d'identifier, sur la façade atlantique à l'ouest du détroit, un faciès particulier désigné sous le nom de Cardial lusitano-marocain.

L'intérêt du schéma construit à partir de KTG et KBS, malgré des imprécisions stratigraphiques qui pourraient subsister, est de pouvoir associer, notamment dans la phase cardiale typique, toutes les composantes du " package " néolithique : céramique et économie de production, avec la domestication attestée d'éléments de la flore et de la faune. En l'état actuel de nos connaissances, la domestication des plantes est documentée dès la première période d'occupation cardiale à KTG (Ballouche, N41). Elle est ensuite associée dans la deuxième période d'occupation avec une faune domestique, moutons et chèvres principalement (Ouchaou, N48), ce qui révèle l'existence d'un véritable système agro-pastoral développé. Pour le moment, ce système n'est pas totalement documenté dans le reste de la péninsule Tingitane. 


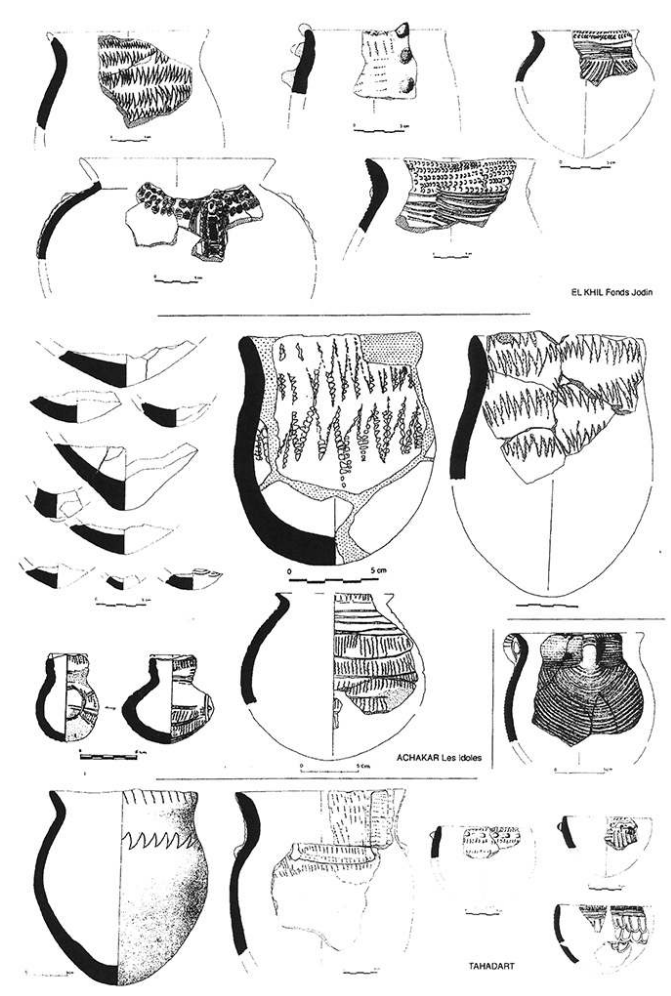

Fig. 4. CÉramique de LA troIsIÈme PHASE CARDIALE de LA PÉNINSULE TINgIRANE (gROUPE LUSITANO-MAROCAIN) : EL KHIL, LES IDOLES ET OUED TAHADART (D'APRÈS EL IDRISSI 2001).

\section{Néolithique ancien du Maroc oriental}

11 Le Néolithique du Maroc Oriental est essentiellement connu à travers les sites des régions de Taza (Hassi Ouenzga) et Al-Hoceima (Ifri Oudadane et Ifri Armas), étudiés dans le cadre de programmes maroco-allemands (coordination A. Mikdad \& J. Eiwanger). Ces connaissances sont ponctuellement complétées par les sites de la région d'Oujda (L. Wengler).

Hassi Ouenzga est le site qui fournit la séquence la mieux étudiée et la mieux datée (Linstädter 2004). Plusieurs faciès céramiques ont été identifiés et datés entre 5600 et 4900 av. J.C. : un faciès typiquement local ayant une céramique à impressions simples, un deuxième faciès de type cardial et un troisième dit de "type oranais ». Ils sont associés à une faune sauvage et, sans trace de domestication des plantes, rapportés à des chasseurs-cueilleurs produisant un outillage lithique comprenant des segments et des lamelles à dos. A ce titre, l'auteur révise sa première appellation de Néolithique ancien et choisit finalement d'attribuer ces ensembles à un Epipaléolithique à poterie (Linstädter 2008).

En revanche, les sites côtiers d'Ifri Oudadane et Ifri Armas (découverts lors des travaux de la «rocade méditerranéenne »), présentent toutes les caractéristiques d'un Néolithique ancien dès la première moitié et le milieu du $6^{\mathrm{e}}$ millénaire av. J.C. : $5.659 \pm$ $39 \mathrm{BC}$ cal. (Linstädter 2010). Le matériel céramique montre une très large variété de décoration imprimée typique du Néolithique ancien : impressions au doigt et à l'aide d'outils indéterminés, ainsi que des décors réalisés à la coquille de Cardium. La poterie «typiquement oranaise " décorée d'incisions entrecroisées est aussi signalée. Les 
animaux domestiques, bœuf, mouton, chèvre et également chien, sont identifiés, accompagnés de l'aurochs, du mouflon à manchettes, du lapin, des gazelles et autres animaux sauvages. La pêche et la collecte des mollusques étaient également pratiquées. Un autre site des iles Chafarinas peut aussi se rattacher à cet ensemble côtier (Bellver Garrido \& Bravo Nieto 2003).

$\mathrm{Au}$ Maroc oriental, les sites de la région d'Oujda (Grotte d'El-Heriga, Oued Isly, Grotte du Rhafas) pourraient assurer la liaison avec la région oranaise (Wengler et al. 1989). Ils fournissent quelques jalons céramiques très proches des poteries incisées de l'Oranais et de Hassi Ouenzga, datés du $5^{\mathrm{e}}$ millénaire av. J.C. La grotte de Guenfouda, située dans les Monts d'Oujda à $30 \mathrm{~km}$ au sud de la ville du même nom, fouillée plus récemment par Aouraghe et al. (2008) semble prometteuse. En l'absence de datation numérique publiée, on peut surtout retenir qu'il s'agirait pour l'instant du site le plus oriental ayant livré de la céramique de type Cardial, associée à de la faune domestique (Caprinés).

\section{Néolithique ancien du Maroc atlantique et central}

Par opposition au Maroc septentrional, la néolithisation dans le reste du pays est très mal documentée. Il y manque, en particulier, les études archéobotaniques et archéozoologiques permettant de préciser le statut économique des sociétés préhistoriques. Dans la tranche de temps concernée, ce sont principalement les sites de la région de Rabat qui fournissent les éléments les plus probants (Daugas et al. 2008); même si, en l'absence d'éléments floristiques et faunistiques, leur attribution au Néolithique pourrait être reconsidérée.

La grotte d'El Harhoura 2 a livré dans un contexte de « Néolithique ancien », une double sépulture datée $\mathrm{du}$ tout début $\mathrm{du} 5 \mathrm{e}$ millénaire av. J.C. La céramique associée, malheureusement remaniée, comprend des tessons présentant «des caractères technologiques et typologiques rapportables au Cardial» (Daugas et al. 2008). Des éléments comparables des grottes des Contrebandiers et El Mnasra permettent aux auteurs de postuler la propagation d'un Cardial de faciès lusitano-marocain durant la première moitié du 5e millénaire vers le sud.

Des sites isolés plus au sud - grotte de Ma Izza près de Oualidia sur la côte ou Toulkine dans le Haut-Atlas de Marrakech - ont aussi livré des céramiques parfois rapprochées du Cardial. Cependant, les données stratigraphiques trop imprécises ou l'absence de datation rendent leur valeur douteuse. De même, un Néolithique typiquement agricole, jusqu'à présent sans céramique, mis en évidence dans le Haouz de Marrakech par l'abondance des houes de pierre taillée dans un outillage lithique spécialisé, serait d'âge bien plus récent (Rodrigue 1985).

\section{Néolithique des franges sahariennes}

Sur les franges sahariennes du Maroc, on aborde une problématique assez différente, à la fois, en raison du contexte culturel et naturel assez distinct des précédents et du type d'archives concernées (sites de plein air, absence de stratigraphie, mauvaise conservation des restes organiques).

Les sites les plus riches sont ceux du Sahara atlantique, en particulier dans la zone Tan Tan-Tarfaya. L'absence de stratigraphie dans la plupart des cas rend très difficiles les 
corrélations. On peut cependant souligner que, sur la tranche temporelle des $6 \mathrm{e} / 5 \mathrm{e}$ millénaires av. J.C., les cultures matérielles sont surtout reconnues à travers un outillage lithique évoquant l'Epipaléolithique. La céramique bien datée est généralement absente. C'est le cas du site 19 de Grébenart (1974), de la dune d'Izriten, qui a livré une sépulture rapprochée d'individus de type Mechtoïde (Petit-Maire 1979), du site Letan (Searight 1998) ou de nos sites TR-3 et TR-15 du plateau de Taoulekt (Onrubia-Pintado et Ballouche 1996). Le mode de vie, fondamentalement de chasseurscueilleurs, s'appuyait à la fois sur les ressources de la mer et sur la faune terrestre, comme la consommation d'œufs d'au $\neg$ truche. Les sites pouvant être rapportés au Néolithique et ayant livré de la céramique se situent plutôt dans le courant du $4 \mathrm{e}$ millénaire av. J.C.

Dans les régions intérieures, les données datées sont encore plus rares. Les premiers travaux d'un programme de coopération franco-marocaine, GENEMAR (pour Genèse du Néolithique marocain), conduit par J.-P. Daugas, F.-Z. Sbihi-Alaoui et M. El Graoui, dans les régions de Figuig et Errachidia, laissaient entrevoir une transition EpipaléolithiqueNéolithique ancien vers 5500 av. J.C. (Daugas et al. 2008).

La richesse de la retombée saharienne des Atlas en sites à gravures rupestres peut aussi fournir quelques éléments de documentation d'un Néolithique pastoral malheureusement mal daté. Le site de l'Adrar n'Metgourine (région d'Akka) est à ce titre exemplaire, avec $68,5 \%$ des sujets gravés représentant des bœufs (Rodrigue 2009). L'industrie lithique étudiée par Grébenart (1995) présente, selon l'auteur, la particularité d'associer des pièces d'aspect paléolithique à un outillage typiquement néolithique et la céramique semble témoigner d'influences à la fois maghrébines et sahariennes.

\section{Recherche d'éléments de comparaison et hypothèses génétiques}

Le Néolithique ancien du Maroc est évidemment loin de constituer un ensemble cohérent et homogène, tant par les caractéristiques typologiques et stylistiques des cultures matérielles que par les systèmes économiques associés. Bien au contraire, la forte différenciation régionale observée peut résulter de deux processus complémentaires : d'une part, l'importance d'influences multiples, normales dans le cas d'un tel carrefour géographique, et d'autre part, la diversité et les spécificités des substrats autochtones préexistants.

Le Néolithique ancien de la péninsule Tingitane, le mieux connu, a fait l'objet de nombreuses hypothèses génétiques. L'hypothèse d'une diffusion du Néolithique depuis l'Espagne sud-orientale par voie maritime via le détroit de Gibraltar a été considérée comme la plus probable (Camps 1974, Roudil 1990). En l'état de nos connaissances actuelles, ce schéma, sans être remis totalement en cause, doit être révisé en y introduisant plus de complexité (Manen et al. 2007, Daugas et al. 2008, Carvalho 2010, Linstädter 2010). Il ne peut, en effet, seul, expliquer la néolithisation de l'ensemble du Maroc septentrional, ni l'existence de styles céramiques particuliers.

Pour El Idrissi (2001), la phase initiale de KTG et KBS peut être considérée comme un jalon dans l'aire reliant la péninsule Tingitane au complexe méditerranéen de la céramique impressionnée dite l'impressa par l'intermédiaire de la zone du Tell algérien. 
Dans les phases principales du Cardial, le registre décoratif de la céramique, permet de la rattacher au Cardial franco-ibérique, au sens large, sinon par filiation directe du moins par différents processus de transfert. Par ailleurs, d'après Linstädter (2008, 2010), le schéma de néolithisation de la péninsule Tingitane, peut partiellement s'étendre plus à l'est sur le littoral méditerranéen du Maroc. Les sites d'Ifri Oudadane et Ifri Armas présentent une évolution comparable dès la première moitié du $6 \mathrm{e}$ millénaire av. J.C. mais, dans ces cas, les contacts auraient eu lieu directement avec la côte andalouse, de façon précoce et sans passage par la voie du détroit de Gibraltar. En effet, le matériel céramique de ces sites diffère nettement, par la simplicité de ses impressions, de celui de la péninsule Tingitane, alors que des parallèles stylistiques semblent exister avec celui des régions de Malaga et Almeria (Linstädter 2010).

Tab. 1 : Datations des principaux sites utilisés dans le texte. En raison des méthodes de calibrations multiples utilisées par les auteurs, nous avons homogénéisé ce tableau en recalibrant toutes les dates radiocarbone (à $1 \sigma$ ) avec le programme CalPal (B. Weninger, Universität zu Köln). Les dates en thermoluminescence (ici en italique) ne sont jamais calibrées mais donnent un âge calendaire moyen.

\begin{tabular}{|c|c|c|c|c|c|}
\hline Site attribution culturelle & $\begin{array}{l}\text { Nr. } \\
\text { Labo }\end{array}$ & $\begin{array}{l}\text { Age }{ }^{14} \mathrm{C} \\
\text { (ou TL) }\end{array}$ & Cal BC & Matériel & Référence \\
\hline KBS Phase initiale & $\begin{array}{l}\text { Rabat } \\
57\end{array}$ & $\begin{array}{l}7589 \quad \pm \\
166\end{array}$ & $\begin{array}{l}6450 \pm \\
170\end{array}$ & charbon & Daugas et al. 2008 \\
\hline KTG Phase initiale & $\begin{array}{l}\text { Rabat } \\
65\end{array}$ & $\begin{array}{ll}7136 & \pm \\
156\end{array}$ & $\begin{array}{l}6020 \pm \\
162\end{array}$ & charbon & Daugas et al. 2008 \\
\hline \multirow{7}{*}{ KTG Cardial } & Cle 128 & $5800 \pm 750$ & $\begin{array}{ll}3820 & \pm \\
750 & \end{array}$ & céramique & Daugas et al. 1998 \\
\hline & Cle 127 & $6350 \pm 600$ & $\begin{array}{ll}4370 & \pm \\
600) & \end{array}$ & céramique & Daugas et al. 1998 \\
\hline & Cle 126 & $6780 \pm 550$ & $\begin{array}{l}4800 \quad \pm \\
550\end{array}$ & céramique & Daugas et al. 1998 \\
\hline & Ly 3821 & $\begin{array}{l}6050 \quad \pm \\
120\end{array}$ & $\begin{array}{l}4990 \pm \\
160\end{array}$ & charbon & Daugas et al. 1989 \\
\hline & Cle 129 & $7200 \pm 750$ & $\begin{array}{ll}5220 & \pm \\
750 & \end{array}$ & céramique & Daugas et al. 1998 \\
\hline & $\begin{array}{l}\text { Ly-971 } \\
\text { OxA }\end{array}$ & $6350 \pm 85$ & $\begin{array}{l}5336 \pm \\
94\end{array}$ & céréale & $\begin{array}{l}\text { Ballouche \& Marnival } \\
2003\end{array}$ \\
\hline & Ly 7288 & $\begin{array}{l}6520 \quad \pm \\
120\end{array}$ & $\begin{array}{l}5470 \pm \\
110\end{array}$ & charbon & Daugas et al. 1989 \\
\hline Tahadart Cardial & UQ 1556 & $\begin{array}{l}5600 \quad \pm \\
200\end{array}$ & $\begin{array}{l}4460 \pm \\
220\end{array}$ & $\begin{array}{l}\text { Coquille } \\
\text { marine }\end{array}$ & Daugas et al. 1998 \\
\hline
\end{tabular}




\begin{tabular}{|c|c|c|c|c|c|}
\hline Grotte des Idoles Cardial & $\begin{array}{l}\text { Gif- } \\
\text { A92332 }\end{array}$ & $5630 \pm 80$ & $\begin{array}{l}4480 \pm \\
90\end{array}$ & charbon & Daugas cr al. 1998 \\
\hline Grotte d'El Khil Cardial & $\begin{array}{l}\text { Rabat } \\
119\end{array}$ & $5720 \pm 114$ & $\begin{array}{l}4580 \pm \\
120\end{array}$ & charbon & Daugas et al. 1998 \\
\hline \multirow{6}{*}{$\begin{array}{l}\text { Ifri Oudadane Néolithique } \\
\text { ancien }\end{array}$} & Erl 9987 & $5756 \pm 49$ & $\begin{array}{ll}4613 \quad \pm \\
62\end{array}$ & charbon & Linstädter 2010 \\
\hline & Erl 9989 & $6053 \pm 50$ & $\begin{array}{ll}4957 \quad \pm \\
70\end{array}$ & charbon & Linstädter 2010 \\
\hline & $\begin{array}{l}\text { KIA } \\
39298\end{array}$ & $6085 \pm 25$ & $\begin{array}{ll}5001 & \pm \\
35\end{array}$ & charbon & Linstädter 2010 \\
\hline & $\begin{array}{l}\text { KIA } \\
39297\end{array}$ & $6155 \pm 30$ & $\begin{array}{l}5124 \pm \\
63\end{array}$ & charbon & Linstâdter 2010 \\
\hline & Erl 9988 & $6175 \pm 50$ & $\begin{array}{l}5131 \pm \\
70\end{array}$ & charbon & Linstädter 2010 \\
\hline & $\begin{array}{l}\text { KIA } \\
39299\end{array}$ & $6400 \pm 90$ & $\begin{array}{l}5377 \pm \\
81\end{array}$ & charbon & Linstädter 2010 \\
\hline \multirow{2}{*}{$\begin{array}{l}\text { Ifri Armas Néolithique } \\
\text { ancien }\end{array}$} & $\begin{array}{l}\text { UBA } \\
8082\end{array}$ & $5989 \pm 33$ & $\begin{array}{ll}4884 \quad \pm \\
44\end{array}$ & $\begin{array}{l}\text { Bos } \\
\text { primigenius }\end{array}$ & Linstädter 2010 \\
\hline & Erl 9996 & $6739 \pm 52$ & $\begin{array}{l}5659 \pm \\
39\end{array}$ & charbon & Linstädter 2010 \\
\hline $\begin{array}{l}\text { Islas Chafarinas Néolithique } \\
\text { ancien }\end{array}$ & $\begin{array}{l}\text { KIA } \\
17373\end{array}$ & $5600 \pm 30$ & $\begin{array}{l}4420 \pm \\
40\end{array}$ & charbon & $\begin{array}{l}\text { Bellver Garrido \& } \\
\text { Bravo Nieto } 2004\end{array}$ \\
\hline \multirow[t]{7}{*}{$\begin{array}{l}\text { Hassi Ouenzga } \\
\text { Epipaléolithique à poterie }\end{array}$} & $\begin{array}{l}\text { Bln } \\
4956\end{array}$ & $6035 \pm 47$ & $\begin{array}{l}4930 \pm \\
70\end{array}$ & charbon & Linstädter 2004 \\
\hline & KIA 437 & $6240 \pm 40$ & $\begin{array}{l}5200 \pm \\
80\end{array}$ & charbon & Linstädter 2004 \\
\hline & $\begin{array}{l}\text { UtC } \\
6185\end{array}$ & $6230 \pm 70$ & $\begin{array}{l}5180 \pm \\
100\end{array}$ & charbon & Linstädter 2004 \\
\hline & KIA 436 & $6270 \pm 40$ & $\begin{array}{l}5260 \pm \\
40\end{array}$ & charbon & Linstädter 2004 \\
\hline & $\begin{array}{l}\text { UtC } \\
6186\end{array}$ & $6378 \pm 44$ & $\begin{array}{l}5380 \pm \\
60\end{array}$ & charbon & Linstädter 2004 \\
\hline & $\begin{array}{l}\text { UtC } \\
6187\end{array}$ & $6540 \pm 50$ & $\begin{array}{l}5510 \pm \\
40\end{array}$ & charbon & Linstädter 2004 \\
\hline & $\begin{array}{l}\text { Bln } \\
4957\end{array}$ & $6611 \pm 40$ & $\begin{array}{l}5560 \pm \\
40\end{array}$ & charbon & Linstädter 2004 \\
\hline
\end{tabular}




\begin{tabular}{|l|l|l|l|l|l|}
\hline \multicolumn{1}{|l|}{\begin{tabular}{|l|l|l|l|l|} 
Bln \\
4913
\end{tabular}} & $6683 \pm 48$ & $\begin{array}{l}5600 \pm \\
40\end{array}$ & charbon & Linstädter 2004 \\
\hline & KIA 434 & $6710 \pm 50$ & $\begin{array}{l}5630 \pm \\
50\end{array}$ & charbon & Linstädter 2004 \\
\hline El Harhoura 2 & UQ 1601 & $\begin{array}{l}5800 \pm \\
150\end{array}$ & $\begin{array}{l}4680 \pm \\
170\end{array}$ & $\begin{array}{l}\text { Coquille } \\
\text { marine }\end{array}$ & Daugas et al. 1998 \\
\cline { 2 - 6 } & Ly 2149 & $5980 \pm 210$ & $\begin{array}{l}4900 \pm \\
260\end{array}$ & Os humain & Daugas et al. 1998 \\
\hline
\end{tabular}

Ainsi, si le modèle de la diffusion cardiale le long des côtes de la Méditerranée occidentale par des «marins » se confirme, il n'est certainement pas de type linéaire. La diffusion ultérieure vers l'intérieur des terres et les modes de réception par des populations autochtones de chasseurs-cueilleurs créent une diversité supplémentaire. Dans les principaux sites du Maroc septentrional ayant livré de la poterie, outre les styles et les décors, les modes de subsistance diffèrent également. Si à KTG et KBS, la céramique cardiale est accompagnée d'abord par la céréaliculture, puis par l'élevage, sur la côte méditerranéenne orientale (Ifri Oudadane, Ifri Armas, Chafarinas) c'est l'élevage qui marque la néolithisation, sans indices de domestication des plantes, alors qu'à l'intérieur (Hassi Ouenzga), les niveaux à poterie du 6e millénaire av. J.C. ne semblent toujours pas connaître d'économie de production. Ces différences peuvent, entre autres, s'expliquer par la diversité des substrats autochtones, d'abord par l'impact plus ou moins fort de leurs structures sociales et économiques, secondairement par les contextes environnementaux locaux.

Par ailleurs, le schéma d'une simple diffusion linéaire doit aussi être nuancé au regard d'autres travaux sur le sud de la péninsule Ibérique (Portugal, Andalousie) où certains traits matériels et techniques de la céramique ( formes en sac», fonds coniques, certaines décorations imprimées, le traitement thermique du silex et l'abondance des armatures lithiques en segment) vont dans le sens d'une influence africaine (Manen et al. 2007, Carvalho 2010). C'est probablement aussi de telles influences réciproques qui permettent d'identifier, plus tard, des affinités suffisamment fortes entre les façades atlantiques marocaine et portugaise pour postuler l'existence d'un Cardial lusitanomarocain distinct d'un faciès alborain (Daugas et al. 2008). Les informations disponibles n'autorisent que des hypothèses, mais il est ainsi permis d'envisager la néolithisation du Maroc septentrional, de l'Atlantique à la Moulouya, comme résultant de l'assemblage d'éléments issus de contacts et d'échanges en directions multiples, souvent transformés et intégrés de façon différenciée à des substrats culturels autochtones qui en assurent une « reformulation» (Manen et al. 2007, Carvalho 2010). C'est ensuite à partir de ce contexte culturel qu'ont pu se dégager les marqueurs d'une évolution vers des styles régionaux ou locaux par acculturation.

La relative méconnaissance des processus comparables dans les régions plus méridionales du Maroc ne permet malheureusement pas d'aller plus loin dans la reconstruction de scénarios plausibles. Il serait particulièrement souhaitable dans les années à venir de pouvoir mieux éclairer les liens que le Néolithique ancien du Maroc a pu entretenir avec le foyer de néolithisation sahélo-saharien. Dans ce sens, l'art 
rupestre des régions atlasiques et sahariennes qui documente, parfois magnifiquement, en l'absence de restes faunistiques, la dimension pastorale d'un Néolithique, encore mal cerné dans ses caractéristiques matérielles et sa chronologie, mérite aussi de nouvelles recherches interdisciplinaires. De plus, par leur position géographique d'interface, c'est certainement aussi sur ces territoires que les traditions méditerranéenne et saharienne ont dû entrer en contact et se modifier. De telles "hybridations" sont assez bien identifiées, plus tard, au Néolithique moyen-récent, dans les styles Achakar ou Skhirat, mais sont encore à rechercher dans le Néolithique ancien.

\section{BIBLIOGRAPHIE}

AOURAGHE H., GAGNEPAin J., HADDOUMI H., El HAMMOUTI K., OUCHAOU B., BAILON S., MESTOUR B., OUJAA A., BOUZOUGGAR A. \& BILLy A., 2008 - «La grotte préhistorique de Guenfouda, Maroc oriental : les premiers résultats (fouilles 2004-2007) », Actes RQM4, Oujda, p. 299-319.

BELLVER GARRIDO J.A. \& BRAVO NIETO A., 2003 - "Una estación neolitíca al aire libre en la Islas Chafarinas : El Zafrín. Primera datación radiocarbónica”, Akros, 2, p. 79-85.

CAMPS G., 1974 - les civilisations préhistoriques de l'Afrique du Nord et du Sahara. Paris, Doin, 366 p.

CARVALHO A.F., 2010 - « Le passage vers l'Atlantique : le processus de néolithisation en Algarve (sud du Portugal) », L'anthropologie, vol. 114, p. 141-178.

DAUGAS J.-P., 2002 - « Le Néolithique du Maroc : pour un modèle d'évolution chronologique et culturelle. Bulletin d'Archéologie Marocaine, t.XIX, p. 135-175.

DAUGAS J.-P., RAYNAL J.-P., BALlOUCHE A., OCChieTti S., PiCheT P., EVIN J., TEXIER J.-P. \& DEBENATH A., 1989 - « Le Néolithique nord-atlantique du Maroc : premier essai de chronologie par le radiocarbone », C. R. Acad. Sci., Paris, sér. II, vol. 308, p. 681-687.

DAUGAS J.-P., EL IDRISSI A., OUSMOI M, FAIN J., MIALLIER D., MONTRET M., SANZELLE S., PILLEYRET., OCCHIETTI S. \& RHODES E.J., 1998 - « Synthèse radio-chronométrique concernant la séquence néolithique au Maroc. Actes du $3^{e}$ Congrès International « 14 C et archéologie », p. 349-353.

DAUGAS J.-P., EL IDRISSi A., BALlOUChE A., MARINVAL P. \& OUCHAOU B., 2008 - « Le Néolithique ancien au Maroc septentrional : données documentaires, sériation typochronologique et hypothèses génétiques. Bulletin de la Société Préhistorique Française, vol. 105, p. 787-812.

EL IDRISSI A., 2001 -. Le Néolithique ancien du Maroc septentrional dans son contexte régional. Thèse, INSAP, Rabat, 2 vol., 415 p.

GILMAN A., 1975 - The Later Prehistory of Tangier, Morocco, Cambridge, Massachussets, Peabody Museum of Archaeology and Ethnology, Harvard University.

GRÉBENART D., 1974 - « Matériaux pour l'étude de l'Épipaléolithique et du Néolithique du littoral atlantique saharien du Maroc ", in : l'Épipaléolithique en Méditerranée, Colloque international Aix-en Provence 1972, p. 151-188. 
GRÉBENART D., 1995 - « Le Néolithique de l'Adrar N'Metgourine, région d'Akka, Maroc », L'homme méditerranéen. Mélanges offerts à Gabriel Camps, Aix-en-Provence, Université de Provence, p. 101-111.

HUYSECOM E. 2006 - « Vers un « Néolithique africain » : concept et apparition », Etudes maliennes, vol. 65 , p. 43-67.

JODINA., 1958 - « Les grottes d'El Khril à Achakar, province de Tanger », Bulletin d'archéologie marocaine, t. III (1958-1959), p. 249-313.

LINSTÄDTER J., 2004 - Zum Frühneolithikum des westlichen Mittelmeerraumes - die Keramik der Fundstelle Hassi Ouenzga, AVAForschungen, vol. 9, 188 p.

LINSTÄDTER J., 2008 - "The Epipalaeolithic-Neolithic-Transition in the Mediterranean region of Northwest-Africa", Quartär, vol. 55, p. 41-62.

LINSTÄDTER J., 2010 - « Recherches récentes sur les sites en grotte du Néolithique ancien dans l'Est marocain », Mémoires de la Société Préhistorique Française, Paris, vol. LI, p. 227-235.

MANEN C., MARCHAND G. \& CARVALHO A.F., 2007 - « Le Néolithique ancien en péninsule Ibérique : vers une nouvelle évaluation du mirage africain?, in: Evin, J. (Ed.), XXVIe Congrès Préhistorique de France. Congrès du Centenaire : un siècle de construction du discours scientifique en Préhistoire, vol. 3 , Paris, Société Préhistorique Française, p. 133-151.

ONRUBIA PINTADO J. \& BALLOUCHE A., 1996 - « Les industries épipaléolithiques de Taoulekt : les sites TR-3 et TR-15 ", in : J. Riser (éd.) Le bassin de Tarfaya (Maroc atlantique, $28^{e}$ Nord). Paléoenvironnements, paléoanthropologie, préhistoire, Paris, L'Harmattan, p. 153-190.

PETIT-MAIRE N., et al. (ed.), 1979 - Le Sahara atlantique à l'Holocène. Paris, AMG (Mémoire XXVIII du CRAPE).

RODRIGUE A., 2009 - L'art rupestre au Maroc : les sites principaux. Des pasteurs du Dra aux métallurgistes de l'Atlas, Paris, L'Harmattan, 191p.

ROUDIL J.L., 1990 - «Cardial et Néolithique ancien ligure dans le Sud-Est de la France », in : Cahen, D., Otte, M. (Eds.), Rubané et Cardial. Néolithique ancien en Europe moyenne, Université de Liège, p. 383-391.

SEARIGHT S., 1998 - « Un campement préhistorique à Tarfaya : Le site Letan », Bulletin d'Archéologie marocaine, vol. XVIII, p. 109-124.

TARRADELLE M., 1955 - « Avance de la primera campaña de excavaciones en Caf Taht el Gar", Tamuda, VI, p. 307-321.

TARRADEELE M., 1957 - «Caf Taht el Gar, cueva neolítica en la región de Tetuán (Marruecos)", Ampurias, vol. 19-20, p. 137-166.

WENGLER L., DELIBRIAS G., MICHEL P. \& VERNET J.-L., 1989 - « Sites néolithiques du Maroc oriental : cadre chronologique, archéologie et milieu naturel ", L'Anthropologie, vol. 93(4), p. 507-534.

\section{INDEX}

Mots-clés : Maroc, Navigation, Néolithique 